hep-ph/0002264

FTUV/00-14

IFIC/00-15

\title{
Bilinear R-parity violating SUSY: Neutrinoless double beta decay in the light of solar and atmospheric neutrino data
}

\author{
M. Hirsch ${ }^{1}$ J. C. Romão ${ }^{2}$ J. W. F. Valle ${ }^{3}$ \\ (a) Instituto de Física Corpuscular - C.S.I.C. \\ Departamento de Física Teòrica, Universitat of València, \\ Edificio Institutos de Paterna, Apartado de Correos 2085 \\ 46071 València \\ (b) Instituto Superior Técnico, Departamento de Física \\ A. Rovisco Pais 1, 1049-001 Lisboa, Portugal
}

PACS: 12.60Jv, 14.60Pq, 23.40-s

\begin{abstract}
Neutrinoless double beta $\left(\beta \beta_{0 \nu}\right)$ decay is considered within bilinear R-parity breaking supersymmetry, including the full one-loop corrections to the neutrinoneutralino mass matrix. Expected rates for $\beta \beta_{0 \nu}$ decay in this model are discussed in light of recent atmospheric and solar neutrino data. We conclude that (a) treelevel calculations for $\beta \beta_{0 \nu}$ decay within the bilinear model are not reliable in the range of parameters preferred by current solar and atmospheric neutrino problems. And (b) if the solar and atmospheric neutrino problems are to be solved within bilinear R-parity violating SUSY the expected rates for $\beta \beta_{0 \nu}$ decay are very low; the effective Majorana neutrino mass at most $0.01 \mathrm{eV}$ and typical values being one order of magnitude lower. Observing $\beta \beta_{0 \nu}$ decay in the next round of experiments therefore would rule out the bilinear R-parity violating supersymmetric model as an explanation for solar and atmospheric neutrino oscillations, as well as any hierarchical scheme for neutrino masses, unless new neutrino interactions are present.
\end{abstract}

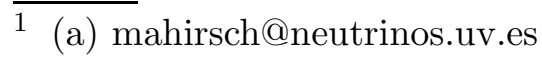

2 (b) romao@gtae3.ist.utl.pt

3 (a) valle@neutrinos.uv.es

Preprint submitted to Elsevier Preprint

27 October 2018 


\section{Introduction}

Neutrino physics has entered a new era recently with the announcement by the SuperKamiokande collaboration of rather conclusive evidence for neutrino oscillations [1] in atmospheric neutrino measurements. This experiment, together with the oscillation interpretation of the long-standing solar neutrino puzzle [2] now provides important information on neutrino masses and mixings and may-be the first look to physics beyond the standard model $[3,4]$.

However, neutrino oscillation experiments, while being extremely valuable, can not answer two fundamental questions in neutrino physics. First, they are only sensitive to mass squared differences and thus can not fix the overall mass scale of neutrinos. And, second, due to the $\mathrm{V}$-A nature of the weak interaction neutrino oscillations can not distinguish in practice between Dirac and Majorana neutrinos. 4 Other experiments on neutrino masses are needed in order to reconstruct the neutrino mass matrix. Neutrinoless double beta decay is a prominent example of such kind of experiments.

Neutrinoless double beta $\left(\beta \beta_{0 \nu}\right)$ decay has for a long time been known as a sensitive probe for physics beyond the standard model (SM). Non-observation of $\beta \beta_{0 \nu}$ decay has been used to derive stringent limits on various extensions of the SM, like, for example, left-right symmetric models [6], leptoquarks [7] and supersymmetry [8,9,11]. However, $\beta \beta_{0 \nu}$ decay has yet to be observed experimentally.

Although there might exist a variety of mechanisms inducing $\beta \beta_{0 \nu}$ decay in gauge theories, one can show that whatever the leading mechanism is at least one of the neutrinos will be a Majorana particle [12]. The observable in $\beta \beta_{0 \nu}$ decay, the effective Majorana neutrino mass, is in general a superposition of different mass eigenstates:

$$
\left\langle m_{\nu}\right\rangle=\sum_{j}^{\prime} U_{e j}^{2} m_{j},
$$

where $U_{e j}$ characterizes the couplings of the mass-eigenstate neutrinos to the electron in the charged current and the prime indicates that the sum runs over light mass eigenstates only. If neutrinos have non-zero mass, also non-zero mixing among them has to be expected, so that in general $\left\langle m_{\nu}\right\rangle$ does not coincide with the electron neutrino mass probed in tritium beta decay.

Currently the most stringent experimental bound [13] gives an upper limit of the order of $\left\langle m_{\nu}\right\rangle \leq \mathcal{O}(0.2-0.5) \mathrm{eV}$. There exist two independent proposals for future experiments which might improve the sensitivity on $\left\langle m_{\nu}\right\rangle$ by up to one order of magnitude or more $[14,15]$.

Here, we concentrate on the calculation of expected rates for $\beta \beta_{0 \nu}$ decay within bilinear R-parity violating (BRPV) SUSY. While $\beta \beta_{0 \nu}$ decay has already been considered in the

$\overline{4}$ The oscillations which are Dirac-Majorana-sensitive must violate lepton number by two units and are helicity suppressed [5] 
literature before within the explicit BRPV SUSY model [9-11], it has so-far only been treated in lowest order of perturbation theory considering the neutrino-neutralino mass matrix only at the tree-level approximation. Here, we take into account the full one-loop corrections to the neutrino-neutralino mass matrix and especially concentrate on those regions in parameter space in which the model can solve simultaneously the solar and atmospheric neutrino problems [16].

We have found that there exist important regions in the parameter space of the model namely those where the BRPV SUSY model can account for the solar neutrino anomaly through matter-enhanced oscillations - where the tree-level estimates for $\beta \beta_{0 \nu}$ decay fail rather badly. Thus the one-loop corrections considered here play an important role in BRPV SUSY. Their inclusion is definitely necessary in order to predict reliably the effective Majorana neutrino mass relevant for $\beta \beta_{0 \nu}$ decay in a way consistent with the results from present oscillation experiments.

This paper is organized as follows. In the next section we set up the notations and discuss the model at tree-level. Then, we outline briefly the extension of the calculation including the one-loop corrections. Further details for these can be found in [17]. Section 4 discusses our numerical results.

\section{Bilinear R-parity violation and neutrino mass at tree-level}

In the following we use conventions such that in the limit were the R-parity violating parameters vanish the usual MSSM notations of refs. [18] are recovered. For the BRPV case see ref. $[19,20]$ for the conventions we adopt. The supersymmetric Lagrangian is specified by the superpotential $W$ given by

$$
W=\varepsilon_{a b}\left[h_{U}^{i j} \widehat{Q}_{i}^{a} \widehat{U}_{j} \widehat{H}_{u}^{b}+h_{D}^{i j} \widehat{Q}_{i}^{b} \widehat{D}_{j} \widehat{H}_{d}^{a}+h_{E}^{i j} \widehat{L}_{i}^{b} \widehat{R}_{j} \widehat{H}_{d}^{a}-\mu \widehat{H}_{d}^{a} \widehat{H}_{u}^{b}+\epsilon_{i} \widehat{L}_{i}^{a} \widehat{H}_{u}^{b}\right]
$$

where $i, j=1,2,3$ are generation indices, $a, b=1,2$ are $S U(2)$ indices, and $\varepsilon$ is a completely antisymmetric $2 \times 2$ matrix, with $\varepsilon_{12}=1$. The symbol "hat" over each letter indicates a superfield, with $\widehat{Q}_{i}, \widehat{L}_{i}, \widehat{H}_{d}$, and $\widehat{H}_{u}$ being $S U(2)$ doublets with hypercharges $\frac{1}{3},-1,-1$, and 1 respectively, and $\widehat{U}, \widehat{D}$, and $\widehat{R}$ being $S U(2)$ singlets with hypercharges $-\frac{4}{3}, \frac{2}{3}$, and 2 respectively. The couplings $h_{U}, h_{D}$ and $h_{E}$ are $3 \times 3$ Yukawa matrices, and $\mu$ and $\epsilon_{i}$ are parameters with units of mass. The last term in eq. (2) is the only $R$-parity violating term.

Supersymmetry breaking is parameterized with a set of soft supersymmetry breaking terms,

$$
\begin{gathered}
V_{\text {soft }}=M_{Q}^{i j 2} \widetilde{Q}_{i}^{a *} \widetilde{Q}_{j}^{a}+M_{U}^{i j 2} \widetilde{U}_{i} \widetilde{U}_{j}^{*}+M_{D}^{i j 2} \widetilde{D}_{i} \widetilde{D}_{j}^{*}+M_{L}^{i j 2} \widetilde{L}_{i}^{a *} \widetilde{L}_{j}^{a}+M_{R}^{i j 2} \widetilde{R}_{i} \widetilde{R}_{j}^{*} \\
+m_{H_{d}}^{2} H_{d}^{a *} H_{d}^{a}+m_{H_{u}}^{2} H_{u}^{a *} H_{u}^{a}-\left[\frac{1}{2} M_{s} \lambda_{s} \lambda_{s}+\frac{1}{2} M \lambda \lambda+\frac{1}{2} M^{\prime} \lambda^{\prime} \lambda^{\prime}+h . c .\right] \\
+\varepsilon_{a b}\left[A_{U}^{i j} \widetilde{Q}_{i}^{a} \widetilde{U}_{j} H_{u}^{b}+A_{D}^{i j} \widetilde{Q}_{i}^{b} \widetilde{D}_{j} H_{d}^{a}+A_{E}^{i j} \widetilde{L}_{i}^{b} \widetilde{R}_{j} H_{d}^{a}-B \mu H_{d}^{a} H_{u}^{b}+B_{i} \epsilon_{i} \widetilde{L}_{i}^{a} H_{u}^{b}\right]
\end{gathered}
$$


and again, the last term in eq. (3) is the only $\mathrm{R}$-parity violating term. The bilinear term in (3) leads in the neutral part of the scalar potential to terms linear in the sneutrino fields. Thus, in general the sneutrino fields acquire VeVs. This in turn leads to mixing between the gaugino and lepton as well as to mixing between the scalar leptons and the Higgs fields $[20,21]$.

For our purposes the most important aspect is the neutrino-neutralino mixing, since it leads at tree-level to one massive neutrino state. In the basis, $\Psi_{0}^{\prime T}=\left(\psi_{L_{1}}^{1}, \psi_{L_{2}}^{1}, \psi_{L_{3}}^{1}\right.$, $\left.-i \lambda^{\prime},-i \lambda_{3}, \psi_{H_{1}}^{1}, \psi_{H_{2}}^{2}\right)$ the neutrino-neutralino mass matrix at tree-level can be written as:

$$
\mathcal{M}_{0}=\left(\begin{array}{cc}
0 & m \\
m^{T} & \mathcal{M}_{\chi^{0}}
\end{array}\right)
$$

Here, the sub-matrix $m$ contains entries from the bilinear $\not R_{p}$ parameters,

$$
m=\left(\begin{array}{cccc}
-\frac{1}{2} g^{\prime} v_{e} & \frac{1}{2} g v_{e} & 0 & \epsilon_{e} \\
-\frac{1}{2} g^{\prime} v_{\mu} & \frac{1}{2} g v_{\mu} & 0 & \epsilon_{\mu} \\
-\frac{1}{2} g^{\prime} v_{\tau} & \frac{1}{2} g v_{\tau} & 0 & \epsilon_{\tau}
\end{array}\right)
$$

where $v_{i}:=\left\langle\tilde{\nu}_{i}\right\rangle$ and $\mathcal{M}_{\chi^{0}}$ is the MSSM neutralino mass matrix, given by,

$$
\mathcal{M}_{\chi^{0}}=\left(\begin{array}{cccc}
M_{1} & 0 & -\frac{1}{2} g^{\prime} v_{d} & \frac{1}{2} g^{\prime} v_{u} \\
0 & M_{2} & \frac{1}{2} g v_{d} & -\frac{1}{2} g v_{u} \\
-\frac{1}{2} g^{\prime} v_{d} & \frac{1}{2} g v_{d} & 0 & -\mu \\
\frac{1}{2} g^{\prime} v_{u} & -\frac{1}{2} g v_{u} & -\mu & 0
\end{array}\right) .
$$

There are two interesting aspects concerning $\mathcal{M}_{0}$. First, $\mathcal{M}_{0}$ has such a texture that at tree-level only one neutrino gets a non-zero mass [22], leaving two massless (but mixed) states in the spectrum. And second, at tree-level the neutrino mass is strictly proportional to the "alignment vector" $|\vec{\Lambda}|^{2}$, where,

$$
\vec{\Lambda}:=\vec{\epsilon} v_{d}+\vec{v} \mu
$$

Thus, at tree-level the individual $\epsilon_{i}$ and $v_{i}$ are not constrained neither by the neutrino mass measurements nor by neutrinoless double beta decay, as long as they are sufficiently aligned. However, we would like to stress (more details below) that this is a pure tree-level result. Once the calculation is improved to one-loop order current experimental hints on solar and atmospheric neutrino oscillations provide rather stringent constraints not only on $\vec{\Lambda}$, but also on the individual BRPV parameters, $\epsilon_{i}$ and $v_{i}$. 
Assuming that $m \ll \mathcal{M}_{0}$ one can find $[9,11]$ a simple formula relating the effective Majorana neutrino mass to the supersymmetric parameters:

$$
\left\langle m_{\nu}\right\rangle \simeq \frac{2}{3} \frac{g^{2} M_{2}}{\operatorname{det}\left(\mathcal{M}_{0}\right)} \Lambda_{e}^{2} .
$$

It has been shown in [11] that within BRPV the contribution from $\left\langle m_{\nu}\right\rangle$ as given above is the dominant source for $\beta \beta_{0 \nu}$ decay. In the following we will concentrate on this BRPV mass mechanism only, improving it by taking into account the one-loop corrections to the neutrino-neutralino mass matrix.

\section{One-loop corrections to the neutrino-neutralino mass matrix}

As we have seen the effective neutrino mass matrix has a projective structure, such that only one neutrino gets a mass at tree-level. As a result for a realistic description of the neutrino spectrum one has to improve the calculation to 1-loop order. 0 A shortened description is given below, for a complete listing of all necessary couplings etc. see ref. [17]. However, most important for the understanding of the importance of the loops is the fact that these contributions explicity break the projectivity of the tree-level mass matrix, incorporating contributions which are proportional to the $\epsilon_{i}$ themselves, as we will show explicitly below. In contrast, as discussed above, the tree-level mass matrix is sensitive only to $\vec{\Lambda}$.

The full neutrino-neutralino mass matrix including the 1-loop corrections is given by

$$
M_{i j}=M_{i j}^{\text {tree }}+\Delta M_{i j}
$$

where $\Delta M_{i j}$ are the 1-loop corrections defined by

$$
\Delta M_{i j}=\frac{1}{2}\left(\Pi_{i j}\left(p_{i}^{2}\right)+\Pi_{i j}\left(p_{j}^{2}\right)-m_{\chi_{i}^{0}} \Sigma_{i j}\left(p_{i}^{2}\right)-m_{\chi_{j}^{0}} \Sigma_{i j}\left(p_{j}^{2}\right)\right)
$$

where $\Sigma_{i j}$ and $\Pi_{i j}$ are self-energies. There are three simple topologies of relevant Feynman diagrams contributing to the neutrino-neutralino mass matrix [17]. ${ }^{6}$. Here, $\overline{D R}$ signifies the minimal dimensional reduction subtraction scheme and $\mu_{R}$ is the renormalization scale. As pointed out in [17] the inclusion of the tadpole diagram is essential in order to obtain gauge invariance of the calculation.

Figure 1 shows the relevant Feynman graphs. Internal particles in the scalar self-energies can be either $(q-\tilde{q})$, (charged scalars-charginos) or (neutral scalars-neutralinos), for the

\footnotetext{
5 With two massless neutrinos, one angle of the neutrino sector of the theory could be rotated away. Thus a discussion of the predictions of the theory for the solar angle is meaningless at tree-level.

6 For a complete description see ref. [17]
} 

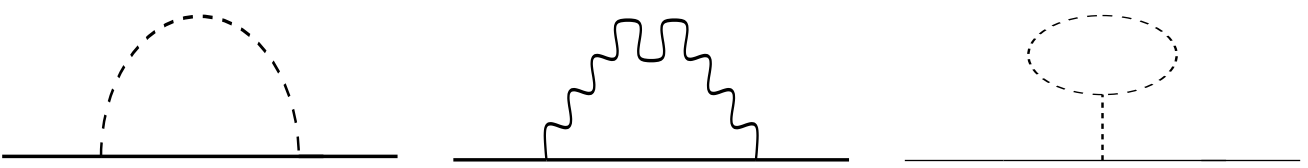

Fig. 1. Simple one-loop topologies contributing to the neutralino-neutrino mass matrix, see text. gauge loops it can either be $\left(W^{ \pm}\right.$-charginos $)$or $\left(Z^{0}\right.$-neutralinos). Which of the loops is most important depends both on parameters and whether one considers the heavy states ("neutralinos") or the light states ("neutrinos"). Here we concentrate on the "neutrino" states. For these only the $(d-\tilde{d})$, (charged scalars-charginos) and ( $W^{ \pm}$-charginos) combinations do indeed contribute. For large values of $\tan \beta$ generally the $(d-\tilde{d})$ loops are most important. We will therefore concentrate on this loop in the following, noting in passing that the basic structure of all the self-energies are the same and can be found by replacing internal masses and couplings correspondingly [17].

It is interesting to note that the tree-level result of neutrino masses being strictly proportional to $|\vec{\Lambda}|^{2}$ is no longer valid once the one-loop contributions are taken into account. This can be shown for example for the down-type squark loops, for which $\Pi_{i j}\left(p_{i}^{2}\right)$ and $\Sigma_{i j}\left(p_{i}^{2}\right)$ are given by,

$$
\begin{gathered}
\Pi_{i j}\left(p_{i}^{2}\right)=\frac{-1}{16 \pi^{2}} \sum_{k, s}\left(\mathcal{O}_{L, j k s}^{n d s} \mathcal{O}_{L, k i s}^{d n s}+\mathcal{O}_{R, j k s}^{n d s} \mathcal{O}_{R, k i s}^{d n s}\right) m_{k} B_{0}\left(m_{i}^{2}, m_{k}^{2}, m_{s}^{2}\right) \\
\Sigma_{i j}\left(p_{i}^{2}\right)=\frac{-1}{16 \pi^{2}} \sum_{k, s}\left(\mathcal{O}_{R, j k s}^{n d s} \mathcal{O}_{L, k i s}^{d n s}+\mathcal{O}_{L, j k s}^{n d s} \mathcal{O}_{R, k i s}^{d n s}\right) B_{1}\left(m_{i}^{2}, m_{k}^{2}, m_{s}^{2}\right)
\end{gathered}
$$

where $B_{0}$ and $B_{1}$ are Passarino-Veltman functions [24], $m_{k}$ and $m_{s}$ are the down-type quark, down-type squark masses and the various $\mathcal{O}$ are neutralino-quark-squark couplings, in our notation given by,

$$
\begin{gathered}
\mathcal{O}_{L i j k}^{\text {dns }}=-\frac{2}{3}\left(\frac{g}{\sqrt{2}}\right) \tan \theta_{W} \mathcal{N}_{j 5}^{*} \mathbf{R}_{k, m+3}^{\tilde{d}^{*}} \mathbf{R}_{\mathbf{R} i, m}^{d}-\left(h_{d}\right)_{m l} \mathbf{R}_{k, m}^{\tilde{d}^{*}} \mathbf{R}_{\mathbf{R} i, l}^{d} \mathcal{N}_{j 7}^{*} \\
\mathcal{O}_{R i j k}^{\text {dns }}=\left(\frac{g}{\sqrt{2}}\right)\left(\mathcal{N}_{j 6}-\frac{1}{3} \tan \theta_{W} \mathcal{N}_{j 5}\right) \mathbf{R}_{k, m}^{\tilde{d}^{*}} \mathbf{R}_{\mathbf{L} m, i}^{* d}-\left(h_{d}^{*}\right)_{m l} \mathbf{R}_{k, l+3}^{\tilde{d}^{*}} \mathbf{R}_{\mathbf{L} m, i}^{* d} \mathcal{N}_{j 7}
\end{gathered}
$$

where the $h_{d}$ denote the down-type Yukawa couplings and $\mathcal{O}_{\text {Lijk }}^{\text {nds }}=\left(\mathcal{O}_{\text {Rjik }}^{\text {dns }}\right)^{*}$ and $\mathcal{O}_{\text {Rijk }}^{\text {nds }}=$ $\left(\mathcal{O}_{L j i k}^{\text {dns }}\right)^{*}$. The rotation matrices $\mathbf{R}^{d}$ and $\mathbf{R}^{\tilde{d}}$ are the ones which diagonalize the quark and squark mass matrices, respectively, while $\mathcal{N}$ diagonalizes the neutralinos/neutrinos.

That terms proportional to $\epsilon_{i}$ survive in eq. (11) is most easily seen assuming the BRPV parameters are small, as suggested by the present indications from solar and atmospheric 
neutrino data. Then one can block-diagonalize the neutrino-neutralino mass matrix perturbatively at tree level in terms of the expansion parameter $\xi=m \cdot \mathcal{M}_{\chi^{0}}^{-1}[23]$ as,

$$
\mathcal{N}^{*}=\left(\begin{array}{cc}
V_{\nu}^{T}\left(1-\frac{1}{2} \xi \xi^{\dagger}\right) & -V_{\nu}^{T} \xi \\
N^{*} \xi^{\dagger} & N^{*}\left(1-\frac{1}{2} \xi^{\dagger} \xi\right)
\end{array}\right)
$$

where $N^{*}$ is the matrix diagonalizing the MSSM part of the neutralino mass matrix and $V_{\nu}^{T}$ describes the mixing of neutrinos among themselves.

The full form for the expansion matrix $\xi$ can be found, for example, in [11]. For our purposes it suffices to state that in the limit $\vec{\Lambda} \equiv 0$ the matrix $V_{\nu}^{T}$ is diagonal, and all elements of $\xi$ vanish except $\xi_{i 3}$, which take the simple form,

$$
\xi_{i 3}=-\frac{\epsilon_{i}}{\mu}
$$

Inserting this result for $\vec{\Lambda} \equiv 0$ and for simplicity considering only $i, j=1,2,3,\left(\sum_{i j}\right.$ vanishes for $i, j=1,2,3$ in this limit) $\Pi_{i j}$ can be written as,

$$
\Pi_{i j}\left(p_{i}^{2}\right)=\frac{-1}{16 \pi^{2}} \frac{\epsilon_{i} \epsilon_{j}}{\mu^{2}} \sum_{k, s}\left(\mathbf{R}_{s, k+3}^{\tilde{d}} \mathbf{R}_{s, k}^{\tilde{d}^{*}}+h . c .\right)\left|\left(h_{d}\right)_{k k}\right|^{2} m_{k} B_{0}\left(m_{i}^{2}, m_{k}^{2}, m_{s}^{2}\right)
$$

where, for simplicity, we have assumed that $h_{d}$ is diagonal. Eq. (17) demonstrates that the entries in $\Pi_{i j}$ in the "neutrino sector" are proportional to $\epsilon_{i} \epsilon_{j}$. This shows explicitly that in the limit where the tree-level neutrino mass vanishes the loop contributions do not and can, potentially, be rather important. Moreover, from this example we can draw two conclusions. First, 1-loop contributions break the projectivity of the mass matrix $\left(m_{i j}^{\text {tree }} \sim \Lambda_{i} \Lambda_{j}\right.$ at tree-level) and thus the degeneracy of the two lightest states is lifted. And, second, the size of the ratio of the 1-loop to the tree-level entries of the mass matrix should be controled mainly by the quantity $|\vec{\epsilon}|^{2} /|\vec{\Lambda}|{ }^{7}$

\section{Numerical results}

In our numerical study we assume unification at a scale $Q=M_{U}$ with standard minimal supergravity boundary conditions,

$$
A_{t}=A_{b}=A_{\tau} \equiv A
$$

$\overline{7}$ In the numerical calculation we have found that this is indeed the case. However, the loops depend also strongly on $\tan \beta$, because large $\tan \beta$ leads to large Yukawa couplings in the down sector, and as shown in eq. (17) the 1-loop entries strongly depend on $h_{d}$. Numerically, variations of other SUSY parameters have been found to be much less important. 


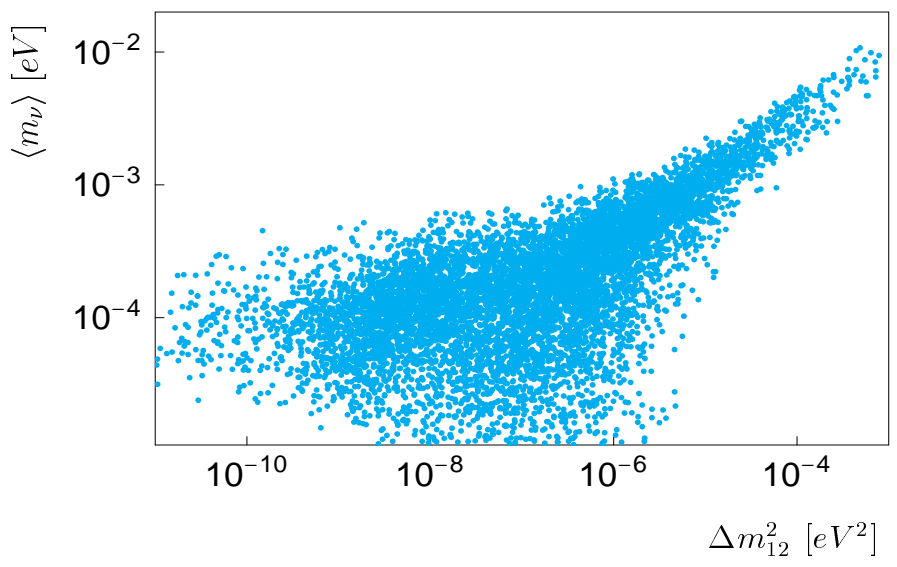

Fig. 2. Effective Majorana neutrino mass as a function of $\Delta m_{12}^{2}$ for data points which have $\sin ^{2}\left(2 \theta_{\text {sol }}\right) \geq 0.6$ and solve the atmospheric neutrino problem.

$$
\begin{aligned}
& B=B_{i}=A-1, \\
& m_{H_{d}}^{2}=m_{H_{u}}^{2}=M_{L_{i}}^{2}=M_{R_{i}}^{2}=m_{0}^{2} \\
& M_{Q_{i}}^{2}=M_{U_{i}}^{2}=M_{D_{i}}^{2}=m_{0}^{2}, \\
& M_{3}=M_{2}=M_{1}=M_{1 / 2} .
\end{aligned}
$$

We run the RGE's from the unification scale $M_{U} \sim 2 \times 10^{16} \mathrm{GeV}$ down to the weak scale, giving random values to the fundamental parameters at the unification scale. We then check that the numerical values obtained from the RGE running correctly break electroweak symmetry. Moreover, we accept only those points for further study, which fulfill phenomenological constraints from negative Higgs and SUSY particle searches at accelerators [25].

Although this procedure is not essential for the calculation of the neutrino masses in the model, it allows us to reduce the number of free parameters considerably and can be viewed as a test for self-consistency of the parameter ranges under consideration.

For the $\not_{p}$ parameters, we use the constraints from solar and atmospheric neutrinos found in $[16,17]$. These two sets of measurements imply that BRPV parameters have to be small, i.e. $|\epsilon|$ and $|\Lambda|$ should be smaller than $\mathcal{O}(\mathrm{GeV})$ and $\mathcal{O}\left(0.2 G e V^{2}\right)$ respectively for typical MSSM parameters smaller than, say $1 \mathrm{TeV}$. $\$$ Moreover, measurements of (or limits on) neutrino angles fix (or yield limits) on ratios of R-parity breaking parameters. Here we summarize these restrictions as follows [17]. The atmospheric neutrino measurements require $\Lambda_{\mu} \simeq \Lambda_{\tau}$, whereas the negative results from the CHOOZ [26] and Palo Verde reactor [27] experiments require that $\Lambda_{e}$ should be smaller than $\Lambda_{e} \leq \mathcal{O}(0.3) \sqrt{\Lambda_{\mu}^{2}+\Lambda_{\tau}^{2}}$. The solar neutrino problem can be either solved with relatively large mixing (LMA-MSW or vacuum oscillations), which implies that all $\epsilon_{i}$ should be similar, or by small mixing

8 Although smaller than usual supersymmetric parameters, such a suppression might be actually expected in scenarios with radiative R-parity breaking ref. [17,19] 


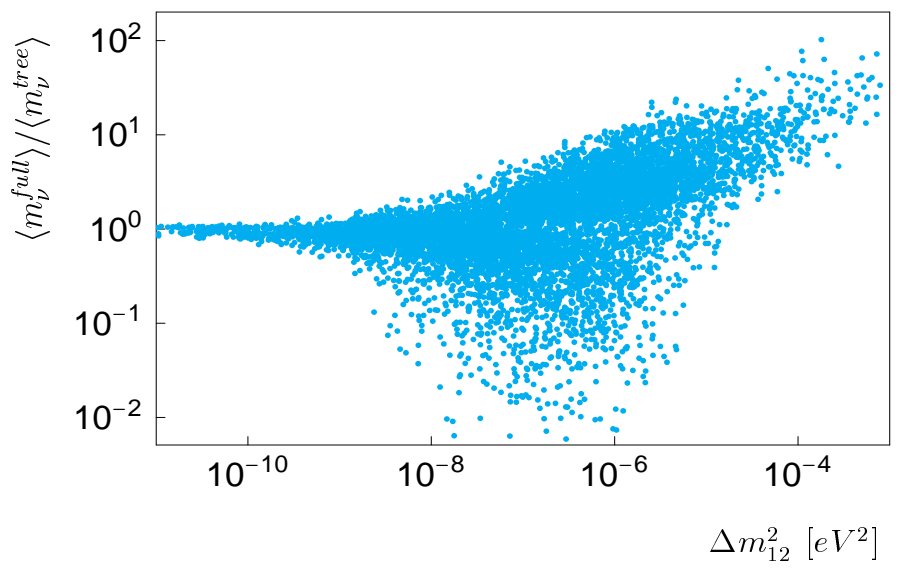

Fig. 3. Ratio of 1-loop corrected effective Majorana neutrino mass to its tree-level value as a function of $\Delta m_{12}^{2}$ for data points which have $\sin ^{2}\left(2 \theta_{\text {sol }}\right) \geq 0.6$ and solve the atmospheric neutrino problem.

(the SMA-MSW solution), the latter implying $\epsilon_{e} \sim($ few $) 10^{-2} \epsilon_{\mu, \tau}$.

We have determined the expected values of $\left\langle m_{\nu}\right\rangle$ as a function of $\Delta m_{12}^{2}$ for about $10^{4}$ calculated points, which solve the atmospheric neutrino problem. Predicted values of $\left\langle m_{\nu}\right\rangle$ are rather small, reaching at most $10^{-2}[\mathrm{eV}]$ for the large mixing solution (LAMSW) of the solar neutrino problem, as can be seen from Fig. (2). For the case of vacuum oscillations $\left\langle m_{\nu}\right\rangle$ will be even much smaller, around $10^{-4}[\mathrm{eV}]$, as seen from the figure.

Let us now discuss the crucial importance of the loop corrections to the neutrino masses in this context. In order to do this we have calculated ratios of $\left\langle m_{\nu}\right\rangle$ including the 1-loop corrections divided by its tree-level value. In figure Fig. (3) we show our results. As can be seen, if $\Delta m_{12}^{2}$ lies in the range required for vacuum (or just-so) oscillations the tree-level and the 1-loop improved $\left\langle m_{\nu}\right\rangle$ are rather similar, whereas for larger $\Delta m_{12}^{2}$ in the MSW range one has a substantial change from the tree-level result. Thus, tree-level calculations of $\left\langle m_{\nu}\right\rangle$ are certainly not accurate in this case, and the 1-loop corrections considered here play an essential role.

Let us now analyze the remaining oscillation possibility to solve the solar neutrino problem, namely the small-angle MSW solution. In this case one finds a suppression in the $\beta \beta_{0 \nu}$ rate, as can be seen in Fig. (4). This result is easy to understand conceptually, as the $\beta \beta_{0 \nu}$ rate must be given in terms of the only $L_{e}$ violating parameters in the model $\Lambda_{e}$ and $\epsilon_{e}$, while $\sin ^{2}\left(2 \theta_{\text {sol }}\right) \rightarrow 0$ as $\Lambda_{e}, \epsilon_{e} \rightarrow 0$.

To close this section we mention that, although we have worked within the framework of a concrete model in which $R_{p}$ constitutes the origin for neutrino mass and mixing, our conclusions are more general. In fact the smallness of effective Majorana neutrino mass $\left\langle m_{\nu}\right\rangle$ holds in any hierarchical model of neutrino mass, of which our bilinear $\not R_{p}$ breaking model is a particular case. Note, that although it is possible in the BRpV model to have two neutrinos nearly degenerate once the 1-loop contributions are included, it is never possible to have all three neutrinos degenerate [17]. Moreover, such points are extremely 


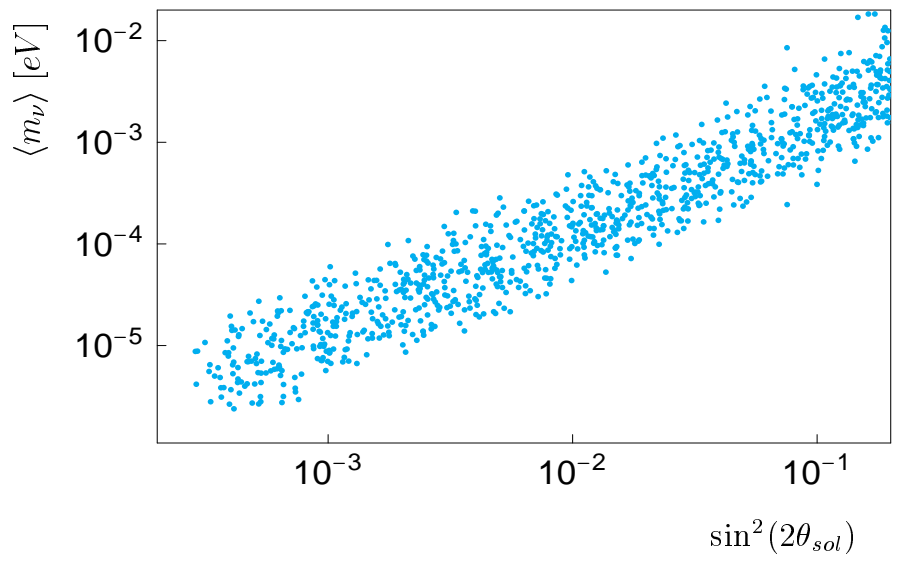

Fig. 4. Expected 1-loop corrected effective Majorana neutrino mass $\left\langle m_{\nu}\right\rangle$ as a function of $\sin ^{2}\left(2 \theta_{\text {sol }}\right)$ for those points which solve the atmospheric neutrino problem.

rare in parameter space and not protected by any symmetry in our model. In hierarchical models, however one expects that the maximum allowed value of $\left\langle m_{\nu}\right\rangle$ (which is achieved for the LA-MSW solution) can be estimated by:

$$
\begin{aligned}
\left\langle m_{\nu}\right\rangle & =\sum_{j}^{\prime} U_{e j}^{2} m_{j} \\
& \sim U_{e 2}^{2} \sqrt{\Delta m_{\text {sol }}^{2}}+U_{e 3}^{2} \sqrt{\Delta m_{a t m}^{2}} \lesssim \frac{1}{2} \sqrt{10^{-4} \mathrm{eV}^{2}}+0.05 \sqrt{10^{-2} \mathrm{eV}^{2}} \sim 0.01 \mathrm{eV}
\end{aligned}
$$

which our numerical results confirm for the BRpV model explicitly. Note, that eq. (19) gives us only an upper bound on $\left\langle m_{\nu}\right\rangle$, but no lower bound and no prediction for $\left\langle m_{\nu}\right\rangle$.

One interesting way to avoid this upper bound is the possibility of neutrinos being closely degenerate in mass. According to our results, this would be a clear indication that BRpV is not the underlying mechanism for generating the solar and atmospheric neutrino masses. Another is if other more exotic mechanisms for solving the neutrino anomalies are entertained, such as flavour changing interactions or decays [28].

\section{Summary}

We have calculated the one-loop corrections to the $\beta \beta_{0 \nu}$ decay observable $\left\langle m_{\nu}\right\rangle$ in bilinear R-parity violating supersymmetry, following the procedure developed in [17]. Since it has been shown in $[16,17]$ that the model is able to solve the solar and atmospheric neutrino problems under certain, relatively simple assumptions, special emphasis has been put in our analysis on those "successful" regions of parameter space.

There are two main results of this study. First, one-loop corrections are important for estimating $\beta \beta_{0 \nu}$ decay rates in bilinear BRPV SUSY. This is due to the fact that the 
model at tree-level has two massless states in the spectrum. This degeneracy is lifted once the one-loop corrections are taken into account. Since tree-level and one-loop masses depend on different combinations of BRPV parameters, which are a priori unknown, the loop corrections can be easily as big as the tree level masses. Especially this is true in those parameter ranges, where the model is able to solve the solar and atmospheric neutrino problems.

Moreover we show that, if bilinear R-parity violating is indeed the solution to the solar and atmospheric neutrino problems, than the expected values of $\left\langle m_{\nu}\right\rangle$ are very small, certainly smaller than $10^{-2} \mathrm{eV}$, and probably even smaller than $10^{-3} \mathrm{eV}$.

Although this conclusion might appear rather discouraging for the experimentalists, we would like to stress that, on the other hand, discovering $\beta \beta_{0 \nu}$ decay at a level significantly larger than $\left\langle m_{\nu}\right\rangle=10^{-2} \mathrm{eV}$ would be sufficient to rule out our model as an explanation for the atmospheric and solar neutrino problems. This conclusion also applies to any hierarchical scheme for neutrino masses. The only possible way this conclusion might be evaded is to consider the presence of exotic neutrino properties, such as flavour changing interactions or decays [28].

\section{Acknowledgement}

This work was supported by DGICYT grant PB98-0693 and by the TMR contract ERBFMRXCT96-0090. M.H. acknowledges support from the European Union's Marie-Curie program under grant No ERBFMBICT983000.

\section{References}

[1] Y. Fukuda et al. [Super-Kamiokande Collaboration], Phys. Rev. Lett. 81, 1562 (1998) hepex/9807003; see also hep-ex/9803006 and hep-ex/9805006

[2] M.B. Smy, "Solar neutrinos with SuperKamiokande," hep-ex/9903034.

[3] For an updated discussion see, M.C. Gonzalez-Garcia, P.C. de Holanda, C. Peña-Garay and J.W.F. Valle, hep-ph/9906469; Nucl. Phys. B, in press.

[4] For an updated discussion see, N. Fornengo, M. C. Gonzalez-Garcia and J. W. F. Valle, hep-ph/0002147 and M.C. Gonzalez-Garcia, H. Nunokawa, O.L. Peres and J. W. F. Valle, Nucl. Phys. B543, 3 (1999)

[5] J. Schechter and J. W. F. Valle, Phys. Rev. D23, 1666 (1981).

[6] M. Hirsch, H.V. Klapdor-Kleingrothaus and O. Panella Phys. Lett. B374 (1996) 7; R.N. Mohapatra, Phys. Rev. D34 (1986) 909

[7] M. Hirsch, H.V. Klapdor-Kleingrothaus and S.G. Kovalenko, Phys. Rev. D54 (1996) R4207

[8] M. Hirsch, H.V. Klapdor-Kleingrothaus and S.G. Kovalenko, Phys. Rev. Lett. 75 (1995) 17; Phys. Rev. D53 (1996) 1329; Phys. Lett. B372 (1996) 181, erratum B381 (1996) 488; A. Faessler, S.G. Kovalenko, F. Simkovic and J. Schwieger, Phys. Rev. Lett. 78 (1997) 183 
[9] A. Faessler, S. Kovalenko, F. Simkovic, Phys.Rev. D57 (1998) 055004 hep-ph/9712535

[10] V. Bednyakov, A. Faessler and S. Kovalenko Phys.Lett. B442 (1998) 203-208

[11] M. Hirsch and J.W.F. Valle, Nucl. Phys. B557 (1999) 60 hep-ph/9812463

[12] J. Schechter and J.W.F. Valle, Phys.Rev. D 25 (1982) 2951

[13] L. Baudis et al., Phys. Rev. Lett. 83, 41 (1999)

[14] H.V. Klapdor-Kleingrothaus, L. Baudis, G. Heusser, B. Majorovits, H. Paes, hepph/9910205

[15] M. Danilov et al., hep-ph/0002003

[16] J.C. Romão, M.A. Díaz, M. Hirsch, W. Porod and J.W.F Valle, hep-ph/9907499 and Phys. Rev. $\mathbf{D}$, in press

[17] M.A. Díaz, M. Hirsch, W. Porod, J.C. Romão and J.W.F Valle, in preparation

[18] H.E. Haber and G.L. Kane, Phys. Rep. 117, 75 (1985); J.F. Gunion and H.E. Haber, Nucl. Phys. B272, 1 (1986); erratum-ibid. B 402, 567 (1993).

[19] M.A. Díaz, J. C. Romão and J. W. F. Valle, Nucl. Phys. B524 (1998) 23 hep-ph/9706315.

[20] A. Akeroyd, M.A. Díaz, J. Ferrandis, M.A. Garcia-Jareño, and Jose W.F. Valle, Nucl. Phys. B529, 3 (1998) hep-ph/9797395].

[21] F. de Campos, M. A. Garcia-Jareno, A. S. Joshipura, J. Rosiek and J. W. F. Valle, Nucl. Phys. B451, 3 (1995) hep-ph/9502237.

[22] A. Santamaria and J. W. F. Valle, Phys. Lett. B195 (1987) 423; Phys. Rev. Lett. 60 (1988) 397; Phys. Rev. D39 (1989) 1780.

[23] J. Schechter and J. W. F. Valle, Phys. Rev. D25, 774 (1982).

[24] G. Passarino and M. Veltman, Nucl. Phys. B160 (1979) 151.

[25] C. Caso et al., "Review of particle physics," Eur. Phys. J. C3, 1 (1998)

[26] M. Apollonio et al., Phys.Lett. B466 (1999) 415, hep-ex/9907037

[27] F. Boehm et al., hep-ex/9912050

[28] N. Fornengo, M. C. Gonzalez-Garcia and J. W. F. Valle, hep-ph/9906539; M. C. GonzalezGarcia et al., Phys. Rev. Lett. 82, 3202 (1999) hep-ph/9809531; V. Barger, J. G. Learned, P. Lipari, M. Lusignoli, S. Pakvasa and T. J. Weiler, Phys. Lett. B462, 109 (1999) hepph/9907421] 